\title{
The Origin of Mass, Spin and Interaction
}

\author{
B.G. Sidharth* \\ International Institute for Applicable Mathematics $\&$ Information Sciences \\ Hyderabad (India) \&s Udine (Italy) \\ B.M. Birla Science Centre, Adarsh Nagar, Hyderabad - 500063 (India)
}

Received 13 March 2009, Accepted 15 April 2009, Published 5 May 2009

\begin{abstract}
We argue that a non commutative geometry at the Compton scale is at the root of mass, Quantum Mechanical spin and QCD and electromagnetic interactions. It also leads to a reconciliation of linearized General Relativity and Quantum Theory.

(C) Electronic Journal of Theoretical Physics. All rights reserved.

Keywords: Quantum Gravity; Noncommutative Geometry; Quantum Fields Interactions; General Relativity

PACS (2008): 04.60.-m; 12.10.-g; 98.80.Qc; 04.60.Pp; 02.40.Gh; 4.20.-q; 04.90.+e; 04.62.+v
\end{abstract}

\section{Introduction}

Modern Fuzzy Spacetime and Quantum Gravity approaches deal with a non differentiable spacetime manifold. In the latter approach there is a minimum spacetime cut off, which, as shown nearly sixty years ago by Snyder leads to what is nowadays called a non commutative geometry, a feature shared by the Fuzzy Spacetime also $[1,2,3,4,5,6]$. The new geometry is given by

$$
\left[d x^{\mu}, d x^{\nu}\right] \approx \beta^{\mu \nu} l^{2} \neq 0
$$

While equation (1) is true for any minimum cut off $l$, it is most interesting and leads to physically meaningful relations including a rationale for the Dirac equation and the underlying Clifford algebra, when $l$ is at the Compton scale (Cf.ref.[3]). In any case given (1), the usual invariant line element,

$$
d s^{2}=g_{\mu \nu} d x^{\mu} d x^{\nu}
$$

\footnotetext{
*birlasc@hd1.vsnl.net.in
} 
has to be written in terms of the symmetric and nonsymmetric combinations for the product of the coordinate differentials. That is the right side of Equation (2) would become

$$
\frac{1}{2} g_{\mu \nu}\left[\left(d x^{\mu} d x^{\nu}+d x^{\nu} d x^{\mu}\right)+\left(d x^{\mu} d x^{\nu}-d x^{\nu} d x^{\mu}\right)\right]
$$

In effect we would have

$$
g_{\mu \nu}=\eta_{\mu \nu}+k h_{\mu \nu}
$$

So the noncommutative geometry introduces an extra term, that is the second term on the right side of (3). It has been shown in detail by the author that (1) or (2) lead to a reconciliation of electromagnetism and gravitation and lead to what may be called an extended gauge formulation $[7,8,9,10]$.

The extra term in (3) leads to an energy momentum like tensor but it must be stressed that its origin is in the non commutative geometry (1). All this of course is being considered at the Compton scale of an elementary particle.

\section{Compton Scale Considerations}

As in the case of General Relativity $[11,12]$, but this time remembering that neither the coordinates nor the derivatives commute we have

$$
\begin{gathered}
\partial_{\lambda} \partial^{\lambda} h^{\mu \nu}-\left(\partial_{\lambda} \partial^{\nu} h^{\mu \lambda}+\partial_{\lambda} \partial^{\mu} h^{\nu \lambda}\right) \\
-\eta^{\mu \nu} \partial_{\lambda} \partial^{\lambda} h+\eta^{\mu \nu} \partial_{\lambda} \partial_{\sigma} h^{\lambda \sigma}=-k T^{\mu \nu}
\end{gathered}
$$

It must be reiterated that the non commutativity of the space coordinates has thrown up the analogue of the energy momentum tensor of General Relativity, viz., $T^{\mu \nu}$. We identify this with the energy momentum tensor.

At this stage, we note that the usual energy momentum tensor is symmetric, this being necessary for the conservation of angular momentum. This condition does not hold in (4), and the circumstance requires some discussion. Let us first consider the usual case with commuting coordinates [13]. Here as is well known, we start with the action integral

$$
S=\int \Lambda\left(q, \frac{\partial q}{\partial x^{2}}\right) d V d t=\frac{1}{c} \int \Lambda d \Omega
$$

In (5) $\Lambda$ is a function of the generalized coordinates $q$ of the system, as also their first derivatives with respect to the space and time coordinates. In our case the $q$ will represent the four potential $A^{\mu}$ (Cf.[13]) as will be seen again in (24). Requiring that (5) should be stationary leads to the usual Euler-Lagrange type equations,

$$
\frac{\partial}{\partial x^{2}} \frac{\partial \Lambda}{\partial q, \imath}-\frac{\partial \Lambda}{\partial q}=0
$$

In (6), the summation convention holds. We also have from first principles

$$
\frac{\partial \Lambda}{\partial x^{\imath}}=\frac{\partial \Lambda}{\partial q} \frac{\partial q}{\partial x^{\imath}}+\frac{\partial \Lambda}{\partial_{q, k}} \frac{\partial_{q, k}}{\partial x^{\imath}}
$$


At this stage we note that in the usual theory we have in (7)

$$
\left\{\frac{\partial q_{, k}}{\partial x^{2}}-\frac{\partial q_{, \imath}}{\partial x^{k}}\right\}=A_{k, \imath}^{l}-A_{\imath, k}^{l}=0
$$

Using (8) it then follows that conservation of angular momentum requires

$$
T^{\imath k}=T^{k \imath}
$$

However in our case the right side of (8) does not vanish due to the non commutativity of the coordinates and the partial derivatives, as will be seen more explicitly in (24). This means that the condition (9) does not hold for non commutative coordinates, and hence (4) does not contradict the conservation of angular momentum. However there is new physics here and this new physics will be seen in equations following from (24): we recover electromagnetism as an effect.

Remembering that $h_{\mu \nu}$ is a small effect, we can use the methods of linearized General Relativity $[11,12]$, to get from (4),

$$
g_{\mu v}=\eta_{\mu v}+h_{\mu v}, h_{\mu v}=\int \frac{4 T_{\mu v}\left(t-\left|\vec{x}-\vec{x}^{\prime}\right|, \vec{x}^{\prime}\right)}{\left|\vec{x}-\vec{x}^{\prime}\right|} d^{3} x^{\prime}
$$

It was shown several years ago in the context of linearized General Relativity, that for distances $\left|\vec{x}-\overrightarrow{x^{\prime}}\right|$ much greater than the distance $\overrightarrow{x^{\prime}}$, that is well outside the Compton wavelength in our case, we can recover from (10) the electromagnetic potential (Cf.ref.[14] and references therein). We will briefly return to this point.

In (10) we use the well known expansions [12]

$$
\begin{gathered}
\bar{T}_{\mu \nu}\left(t-\left|x-x^{\prime}\right|, x^{\prime}\right)=\sum_{n=0}^{\infty} \frac{1}{n}\left[\frac{\partial^{n}}{\partial t^{n}} \bar{T}_{\mu \nu}\left(t-r, x^{\prime}\right)\right]\left(r-\left|x-x^{\prime}\right|\right)^{n}, \\
r-\left|x-x^{\prime}\right|=x^{j}\left(\frac{x^{j^{\prime}}}{r}\right)+\frac{1}{2} \frac{x^{j} x^{k}}{r}\left(\frac{x^{j^{\prime}} x^{k^{\prime}}-r^{\prime 2} \delta_{j k}}{r^{2}}\right)+\cdots, \\
\frac{1}{\left|x-x^{\prime}\right|}=\frac{1}{r}+\frac{x^{j}}{r^{2}} \frac{x^{j^{\prime}}}{r}+\frac{1}{2} \frac{x^{j} x^{k}}{r^{3}} \frac{\left(3 x^{j^{\prime}} x^{k^{\prime}}-r^{\prime 2} \delta_{j k}\right)}{r^{2}}+\cdots,
\end{gathered}
$$

where $r \equiv|\vec{x}|$. We note that

$$
r=|\vec{x}| \sim l
$$

where $l$ is of the order of the Compton wavelength. So the expansion of the integral in (10) now gives using (12) and (13),

$$
\frac{T}{r}+T^{\prime} \cdot \frac{1}{r}\left(r-\left|x-x^{\prime}\right|\right)+\frac{1}{2} T^{\prime \prime} \frac{\left(r-\left|x-x^{\prime}\right|\right)}{r}
$$

where primes denote the derivatives and we have dropped the superscripts for the moment. Denoting $\left(r-\left|x-x^{\prime}\right|\right) \equiv r^{\prime}$, where $0 \leq r^{\prime} \leq r$, we can write

$$
\left\langle r^{\prime}\right\rangle \approx \gamma r \text { where } \gamma \sim 0(1)
$$


Finally the expansion gives on the use of (15) and (16), the expression

$$
\frac{T}{r}+\gamma T^{\prime}+\frac{\gamma^{2}}{2} T^{\prime \prime} \cdot r
$$

That is we have, from (10) and (17),

$$
\begin{array}{r}
h_{\mu v}=4 \int \frac{T_{\mu v}\left(t, \vec{x}^{\prime}\right)}{\left|\vec{x}-\vec{x}^{\prime}\right|} d^{3} x^{\prime}+(\text { terms independent of } \vec{x})+2 \\
\int \frac{d^{2}}{d t^{2}} T_{\mu v}\left(t, \vec{x}^{\prime}\right) \cdot\left|\vec{x}-\vec{x}^{\prime}\right| d^{3} x^{\prime}+0\left(\left|\vec{x}-\vec{x}^{\prime}\right|^{2}\right)
\end{array}
$$

The first term gives on the use of (17), a Coulombic $\frac{\alpha}{r}$ type interaction except that the coefficient $\alpha$ is of much greater magnitude as compared to the gravitational or electromagnetic case, because in the expansion (12) and (13) all terms are of comparable order in view of (14). The second term on the right side of (18) is of no dynamical significance as it is independent of $\vec{x}$. The third term however is of the form constant $\times r$. That is the potential (18) is exactly of the form of the QCD potential [15]

$$
-\frac{\alpha}{r}+\beta r
$$

In (19) $\alpha$ is of the order of the mass of the particle as follows from (18) and the fact that $T^{\mu \nu}$ is the energy omentum tensor given by

$$
T^{\mu \nu}=\rho u^{\mu} u^{\nu}
$$

where in (20), remembering that (14) holds, that is we are at the Compton scale, $u^{2} \sim c$. We now deduce two relations which can be deduced directly from the theory of the Dirac equation [16]. We do it here to show the continuity of the above theme. Remembering that from (1), we are within a sphere of radius given by the Compton length where the velocities equal that of light, as noted above, we have equations

$$
\begin{gathered}
\left|\frac{d u_{v}}{d t}\right|=\left|u_{v}\right| \omega \\
\omega=\frac{\left|u_{v}\right|}{R}=\frac{2 m c^{2}}{\hbar}
\end{gathered}
$$

Alternatively as remarked, we can get (21) from the theory of the Dirac equation itself [16], viz.,

$$
\imath \hbar \frac{d}{d t}\left(u_{\imath}\right)=-2 m c^{2}\left(u_{\imath}\right)
$$

Using (20), (21) and (22) we get

$$
\frac{d^{2}}{d t^{2}} T^{\mu v}=4 \rho u^{\mu} u^{v} \omega^{2}=4 \omega^{2} T^{\mu v}
$$

(Equation (23) too is obtained in the Dirac theory (loc.cit)). Whence, as can be easily verified, $\alpha$ and $\beta$ in (19) have the correct values required for the QCD potential (Cf. also 
[14]). (Alternatively $\beta r$ itself can be obtained, as in the usual theory by a comparison with the Regge angular momentum mass relation: It is in fact the constant string tension like potential which gives quark confinement and its value is as in the usual theory [17]). Let us return to the considerations which lead via a non commutative geometry to an energy momentum tensor in (4). We can obtain from here the origin of mass and spin itself, for we have as is well known (Cf.ref.[12])

$$
m=\int T^{00} d^{3} x
$$

and via

$$
S_{k}=\int \epsilon_{k l m} x^{l} T^{m 0} d^{3} x
$$

the equation

$$
S_{k}=c<x^{l}>\int \rho d^{3} x
$$

While $m$ above can be immediately and consistently identified with the mass, the last equation gives the Quantum Mechanical spin if we remember that we are working at the Compton scale so that

$$
\left\langle x^{l}\right\rangle=\frac{\hbar}{2 m c} .
$$

Returning to the considerations in (1) to (4) it follows that (Cf.ref.[7])

$$
\frac{\partial}{\partial x^{\lambda}} \frac{\partial}{\partial x^{\mu}}-\frac{\partial}{\partial x^{\mu}} \frac{\partial}{\partial x^{\lambda}} \text { goes over to } \frac{\partial}{\partial x^{\lambda}} \Gamma_{\mu \nu}^{\nu}-\frac{\partial}{\partial x^{\mu}} \Gamma_{\lambda \nu}^{\nu}
$$

Normally in conventional theory the right side of (24) would vanish. Let us designate this non vanishing part on the right by

$$
\frac{e}{c \hbar} F^{\mu \lambda}
$$

We have shown here that the non commutativity in momentum components leads to an effect that can be identified with electromagnetism and in fact from expression (25) we have

$$
A^{\mu}=\hbar \Gamma_{\nu}^{\mu \nu}
$$

where $A_{\mu} \equiv q$, which we encountered in (6), as noted can be identified with the electromagnetic four potential and the Coulomb law deduced for $\left|\vec{x}-\vec{x}^{\prime}\right|$ in (10) much greater than $\left|\vec{x}^{\prime}\right|$ that is well outside the Compton scale (Cf.ref.[3] and also ref. [14]). Indeed we have referred to this in the discussion after (4). It must be mentioned that despite non commutativity, we are using as an approximation the usual continuous partial derivatives, though these latter do not commute amongst themselves now. This facilitates the analysis and brings out the physical effects. In any case as can be seen from (1), the effects are of the order $l^{2}$.

To see this in the light of the usual gauge invariant minimum coupling (Cf.ref.[14]), we start with the effect of an infinitesimal parallel displacement of a vector in this non commutative geometry,

$$
\delta a^{\sigma}=-\Gamma_{\mu \nu}^{\sigma} a^{\mu} d x^{\nu}
$$


As is well known, (27) represents the effect due to the curvature and non integrable nature of space - in a flat space, the right side would vanish. Considering the partial derivatives with respect to the $\mu^{\text {th }}$ coordinate, this would mean that, due to (27)

$$
\frac{\partial a^{\sigma}}{\partial x^{\mu}} \rightarrow \frac{\partial a^{\sigma}}{\partial x^{\mu}}-\Gamma_{\mu \nu}^{\sigma} a^{\nu}
$$

Letting $a^{\mu}=\partial^{\mu} \phi$, we have, from (28)

$$
\begin{gathered}
D_{\mu \nu} \equiv \partial_{\nu} \partial^{\mu} \rightarrow D_{\mu \nu}^{\prime} \equiv \partial_{\nu} \partial^{\mu}-\Gamma_{\lambda \nu}^{\mu} \partial^{\lambda} \\
=D_{\mu}-\Gamma_{\lambda \nu}^{\mu} \partial^{\lambda}
\end{gathered}
$$

Now we can also write

$$
D_{\mu \nu}=\left(\partial^{\mu}-\Gamma_{\lambda \lambda}^{\mu}\right)\left(\partial_{\nu}-\Gamma_{\lambda \nu}^{\lambda}\right)+\partial^{\mu} \Gamma_{\lambda \nu}^{\lambda}+\Gamma_{\lambda \lambda}^{\mu} \partial_{\nu}
$$

So we get

$$
D_{\mu \nu}-\Gamma_{\lambda \lambda}^{\mu} \partial_{\nu}=\left(p^{\mu}\right)\left(p_{\nu}\right)
$$

where

$$
p^{\mu} \equiv \partial^{\mu}-\Gamma_{\lambda \lambda}^{\mu}
$$

Or,

$$
D_{\mu \mu}-\Gamma_{\lambda \lambda}^{\mu} \partial_{\mu}=\left(p^{\mu}\right)\left(p_{\mu}\right)
$$

Further we have

$$
D_{\mu \mu}^{\prime}=D_{\mu \mu}-\Gamma_{\lambda \mu}^{\mu} \partial^{\lambda}
$$

Thus, (29) gives, finally,

$$
D_{\mu \nu}^{\prime}=\left(p_{\mu}\right)\left(p_{\nu}\right)
$$

That is we have

$$
\frac{\partial}{\partial x^{\mu}} \rightarrow \frac{\partial}{\partial x^{\mu}}-\Gamma_{\mu \nu}^{\nu}
$$

Comparison with (26) establishes the required identification.

It is quite remarkable that equation (26) is mathematically identical to Weyl's unified formulation, though this was not originally acceptable because of the ad hoc insertion of the electromagnetic potential. Here in our case it is a consequence of the geometry - the noncommutative geometry (Cf.refs.[14] and [18] for a detailed discussion).

It was also described in detail how in the usual commutative spacetime the Dirac spinorial wave functions conceal the noncommutative character (1) [3].

Indeed we can verify all these considerations in a simple way as follows:

First let us consider the usual spacetime, in which the Dirac wave function is given by

$$
\psi=\left(\begin{array}{l}
\chi \\
\Theta
\end{array}\right),
$$


where $\chi$ and $\Theta$ are two component spinors. It is well known that under reflection while the so called positive energy spinor $\Theta$ behaves normally, on the contrary $\chi \rightarrow-\chi, \chi$ being the so called negative energy spinor which comes into play at the Compton scale [19]. That is, space is doubly connected. Because of this property as shown in detail [8], there is now a covariant derivative given by, in units, $\hbar=c=1$,

$$
\frac{\partial \chi}{\partial x^{\mu}} \rightarrow\left[\frac{\partial}{\partial x^{\mu}}-n A^{\mu}\right] \chi
$$

where

$$
A^{\mu}=\Gamma_{\sigma}^{\mu \sigma}=\frac{\partial}{\partial x^{\mu}} \log (\sqrt{|g|})
$$

$\Gamma$ denoting the Christoffel symbols.

$A^{\mu}$ in (31)is now identified with the electromagnetic potential, exactly as in Weyl's theory except that now, $A^{\mu}$ arises from the bi spinorial character of the Dirac wave function or the double connectivity of spacetime. In other words, we return to (26) via an alternative route.

What all this means is that the so called ad hoc feature in Weyl's unification theory is really symptomatic of the underlying noncommutative spacetime geometry (1). Given (1) (or (3)) we get both gravitation and electromagnetism in a unified picture, because both are now the consequence of spacetime geometry. We could think that gravitation arises from the symmetric part of the metric tensor (which indeed is the only term if $0\left(l^{2}\right)$ is neglected) and electromagnetism from the antisymmetric part (which manifests itself as an $0\left(l^{2}\right)$ effect). It is also to be stressed that in this formulation, we are working with noncommutative effects at the Compton scale, this being true for the Weyl like formulation also.

\section{Discussion}

The Compton scale comes as a Quantum Mechanical effect, within which we have zitterbewegung effects and a breakdown of Causal Physics [16]. Indeed Dirac had noted this aspect in connection with two difficulties with his electron equation. Firstly the speed of the electron turns out to be the velocity of light. Secondly the position coordinates become complex or non Hermitian. His explanation was that in Quantum Theory we cannot go down to arbitrarily small spacetime intervals, for the Heisenberg Uncertainty Principle would then imply arbitrarily large momenta and energies. So Quantum Mechanical measurements are an average over intervals of the order of the Compton scale. Once this is done, we recover meaningful physics. All this has been studied afresh by the author more recently, in the context of a non differentiable spacetime and noncommutative geometry [20].

Weinberg also notices the non physical aspect of the Compton scale [21]. Starting with the usual light cone of Special Relativity and the inversion of the time order of events, he goes on to add, and we quote at a little length and comment upon it, "Although the relativity of temporal order raises no problems for classical physics, it plays a profound 
role in quantum theories. The uncertainty principle tells us that when we specify that a particle is at position $x_{1}$ at time $t_{1}$, we cannot also define its velocity precisely. In consequence there is a certain chance of a particle getting from $x_{1}$ to $x_{2}$ even if $x_{1}-x_{2}$ is spacelike, that is, $\left|x_{1}-x_{2}\right|>\left|x_{1}^{0}-x_{2}^{0}\right|$. To be more precise, the probability of a particle reaching $x_{2}$ if it starts at $x_{1}$ is nonnegligible as long as

$$
\left(x_{1}-x_{2}\right)^{2}-\left(x_{1}^{0}-x_{2}^{0}\right)^{2} \leq \frac{\hbar^{2}}{m^{2}}
$$

where $\hbar$ is Planck's constant (divided by $2 \pi$ ) and $m$ is the particle mass. (Such space-time intervals are very small even for elementary particle masses; for instance, if $m$ is the mass of a proton then $\hbar / m=2 \times 10^{-14} \mathrm{~cm}$ or in time units $6 \times 10^{-25} \mathrm{sec}$. Recall that in our units $1 \mathrm{sec}=3 \times 10^{10} \mathrm{~cm}$.) We are thus faced again with our paradox; if one observer sees a particle emitted at $x_{1}$, and absorbed at $x_{2}$, and if $\left(x_{1}-x_{2}\right)^{2}-\left(x_{1}^{0}-x_{2}^{0}\right)^{2}$ is positive (but less than or $=\hbar^{2} / m^{2}$ ), then a second observer may see the particle absorbed at $x_{2}$ at a time $t_{2}$ before the time $t_{1}$ it is emitted at $x_{1}$.

"There is only one known way out of this paradox. The second observer must see a particle emitted at $x_{2}$ and absorbed at $x_{1}$. But in general the particle seen by the second observer will then necessarily be different from that seen by the first. For instance, if the first observer sees a proton turn into a neutron and a positive pi-meson at $x_{1}$ and then sees the pi-meson and some other neutron turn into a proton at $x_{2}$, then the second observer must see the neutron at $x_{2}$ turn into a proton and a particle of negative charge, which is then absorbed by a proton at $x_{1}$ that turns into a neutron. Since mass is a Lorentz invariant, the mass of the negative particle seen by the second observer will be equal to that of the positive pi-meson seen by the first observer. There is such a particle, called a negative pi-meson, and it does indeed have the same mass as the positive pimeson. This reasoning leads us to the conclusion that for every type of charged particle there is an oppositely charged particle of equal mass, called its antiparticle. Note that this conclusion does not obtain in nonrelativistic quantum mechanics or in relativistic classical mechanics; it is only in relativistic quantum mechanics that antiparticles are a necessity. And it is the existence of antiparticles that leads to the characteristic feature of relativistic quantum dynamics, that given enough energy we can create arbitrary numbers of particles and their antiparticles."

We note however that there is a nuance here which distinguishes Weinberg's explanation from that of Dirac. In Weinberg's analysis, one observer sees only protons at $x_{1}$ and $x_{2}$, whereas the other observer sees only neutrons at $x_{1}$ and $x_{2}$ while in between, the first observer sees a positively charged pion and the second observer a negatively charged pion. We remark that in Weinberg's explanation which is in the spirit of the Feynman-Stuckleberg diagrams there is no charge conservation, though the Baryon number is conserved. The explanation for this is to be found in the considerations leading from (10) to (19) - within the Compton scale we have the QCD interactions - electromagnetic interaction is outside the Compton scale [22].

Our analysis uses the Compton length (and time) as the fundamental parameter. It may be added that there is a close parallel between the above considerations and the orig- 
inal Dirac monopole theory: in the latter it is the nodal singularity that gives rise to magnetism, while in the former, the multiply connected nature of space (or non commutativity) gives rise to electromagnetism. This has been discussed in [23]. So too, it may be mentioned that the considerations in equations (21), (22) and (23) are connected with Dirac's membrane (and more recently and generally the p-brane) theory [24] - though Dirac himself approached the membrane problem from a different route.

Finally, it may be pointed out that Einstein himself always disliked the energy momentum tensor in his General Relativistic equation [25] as it was mechanical and non geometric! Pleasingly, in the above formulation, this term has a geometric origin - albeit, a non commutative geometry which also provides a unified description of linearized General Relativity and Quantum Mechanics.

\section{Acknowledgement}

The author is grateful to the referee for the useful comments.

\section{References}

[1] Snyder, H.S., Physical Review, Vol.72, No.1, July 1 1947, p.68-71.

[2] Amati, D., in "Sakharov Memorial Lectures", Eds. L.V. Kaddysh and N.Y. Feinberg, Nova Science, New York, 1992, pp.455ff.

[3] Sidharth, B.G., "The Universe of Fluctuations", Springer, Dordrecht, 2005.

[4] Kempf, A., in "From the Planck length to the Hubble radius", Ed. A. Zichichi, World Scientific, Singapore, 2000, pp.613ff.

[5] Madore, J., "An Introduction to Non-Commutative Differential Geometry", Cambridge University Press, Cambridge, 1995.

[6] Madore, J., Class.Quantum Grav. 9, 1992, p.69-87.

[7] Sidharth, B.G., Nuovo Cimento B 116B (6) 2001, p.4ff. 1

[8] Sidharth, B.G., Nuovo Cimento B 117B (6) 2002, pp.703ff.

[9] Sidharth, B.G., Int.J.Mod.Phys.E., 14 (2) 2005, $215 \mathrm{ff}$.

[10] Sidharth, B.G., "Gravitation From a Gauge Like Formulation", to appear in Annales Fondation L. De Broglie.

[11] Ohanian, C.H., and Ruffini, R., "Gravitation and Spacetime", New York, 1994, pp.64ff.

[12] Misner, C.W., Thorne, K.S., and Wheeler, J.A., "Gravitation", W.H. Freeman, San Francisco, 1973, pp.819ff.

[13] Landau, L.D., and Lifshitz, E.M., "The Classical Theory of Fields", Pergamon Press, Oxford, 1975, pp.77ff.

[14] Sidharth, B.G., "Chaotic Universe: From the Planck to the Hubble Scale", Nova Science, New York, 2001. 
[15] Lee, T.D., "Particle Physics and Introduction to Field Theory", Harwood Academic, 1981, pp.391ff.

[16] Dirac, P.A.M., "The Principles of Quantum Mechanics", Clarendon Press, Oxford, 1958, pp.4ff, pp.253ff.

[17] Smit, J., "Quantum Fields on a Lattice", Cambridge University Press, 2002, pp.2ff.

[18] Sidharth, B.G., Annales Fondation L. De Broglie, 29 (3), 2004, 1.

[19] Bjorken, J.D., and Drell, S.D., "Relativistic Quantum Mechanics", Mc-Graw Hill, New York, 1964, p.39.

[20] Sidharth, B.G., Found.Phys.Lett., 15 (5), 2002, 501ff.

[21] Weinberg, S., "Gravitation and Cosmology", John Wiley \& Sons, New York, 1972, p.61ff.

[22] Sidharth, B.G., "Planck Scale to Compton Scale" to appear in Int.J.Mod.Phys.E.

[23] Sidharth, B.G., Nuovo Cimento B, 118B (1), 2003, pp.35-40.

[24] Sidharth, B.G., Electromagnetic Phenomena, Vol.6, No.1 (16), 2006.

[25] Nambu, Y., "Quarks - Frontiers in Elementary Particle Physics", World Scientific, Singapore, 1981, p.212. 